\title{
ToF-SIMS Investigations of Tip-Surface Chemical Interactions in Atomic Force Microscopy on a Combined AFM/ToF-SIMS Platform
}

\author{
Chance C. Brown ${ }^{1,2}$, Anton V. Ievlev ${ }^{1,3}$, Petro Maksymovych ${ }^{1,3}$, Sergei V. Kalinin ${ }^{1,3}$, Olga S. \\ Ovchinnikova ${ }^{1,3}$ \\ 1. Center for Nanophase Materials Sciences, Oak Ridge National Laboratory, Oak Ridge, TN \\ 2. Bredesen Center for Interdisciplinary Research and Graduate Education, University of Tennessee, \\ Knoxville, TN \\ ${ }^{3 .}$ Institute for Functional Imaging of Materials, Oak Ridge National Laboratory, Oak Ridge, TN
}

Atomic force microscopy (AFM) has become one of the premier tools of nanoscale surface characterization over the last 20 years. The technique is used in a variety of industries to understand topological and functional (electrical, mechanical, magnetic) properties of the material surfaces, but has the disadvantage of being chemically insensitive [1, 2]. However, this problem can be solved by combination of AFM with one of mass spectrometric techniques. Here, we utilized combined Time-ofFlight Secondary Ion Mass Spectrometry (ToF-SIMS) with AFM to understand chemical effects in the AFM tip-surface junction during contact mode scanning. Electrochemical processes are important in materials, for instance it can significantly affect spontaneous polarization behavior in ferroelectrics [3] and the combined ToF-SIMS/AFM system can provide insight to such phenomena. Previous studies using this system have shown that SPM tip-induced polarization switching in ferroelectrics can cause changes in surface and bulk chemistry [4].

To understand the chemical effects of AFM on samples, systematic changes in tip parameters (contact force, scanning velocity, applied electrical bias) were employed while scanning in contact AFM mode $\mathrm{PbZr}_{0.2} \mathrm{Ti}_{0.8} \mathrm{O}_{3}$ (PZT) and $\mathrm{SrTiO}_{3}$ (STO) films. The samples were cleaned in-situ using an oxygen ion sputtering gun to eliminate adsorption surface layer influences. After scanning, local changes of surface chemistry were inspected using ToF-SIMS.

ToF-SIMS investigation of the surface revealed no changes in the underlying sample chemistry. Multivariate analysis of data show layers of Si deposition in scanned region, which is confirmed using a high spectral resolution ToF-SIMS imaging (Fig. 1). Tip parameters were varied while scanning $10 \mu \mathrm{m}$ squares and show that scanning speed is the primary parameter controlling Si deposition (Fig. 2), which shows chemical interaction at the tip-surface junction drives this phenomenon.

This study shows first time observations of chemical effects that occur at the tip-surface junction using a combined AFM/ToF-SIMS system [5].

\section{References:}

[1] Binning G.K, Phys. Scr., T19A (1987), p.53

[2] Kalinin S.V. et.al, Rep. Prog Phys 73 (2010), p. 056502.

[3] Wang R.V. et.al, Phys. Rev. Lett 102 (2009), p. 047601.

[4] Ievlev, A.V.et.al, ACS Applied Materials \& Interfaces 8(43) (2016), p. 29588.

[5] Research supported by Oak Ridge National Laboratory's Center for Nanophase Materials Sciences

(CNMS), which is a U.S. Department of Energy, Office of Science User Facility. 
(a)

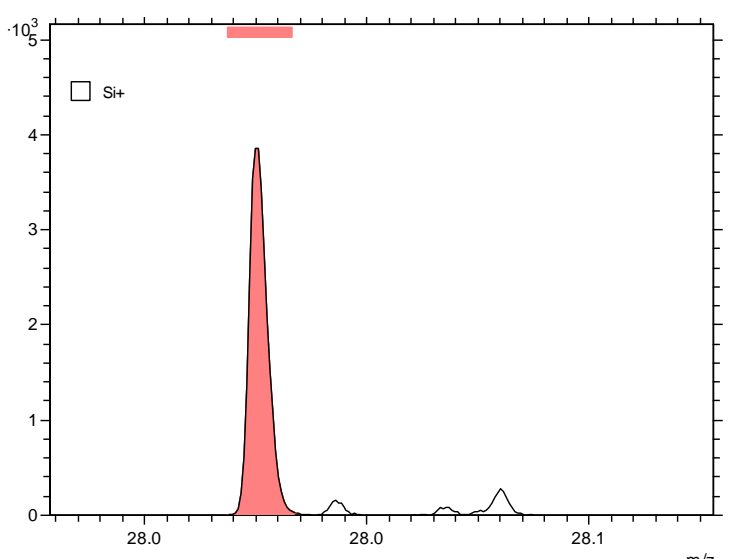

(b)

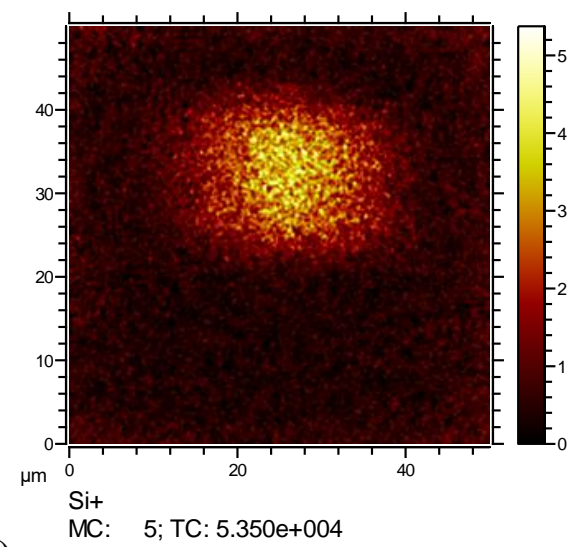

Figure 1. (a) High resolution mass spectra showing Si peak (left) and (b) map of $\mathrm{Si}^{+}$spatial distribution inside $10 \mu \mathrm{m}$ scanned region.

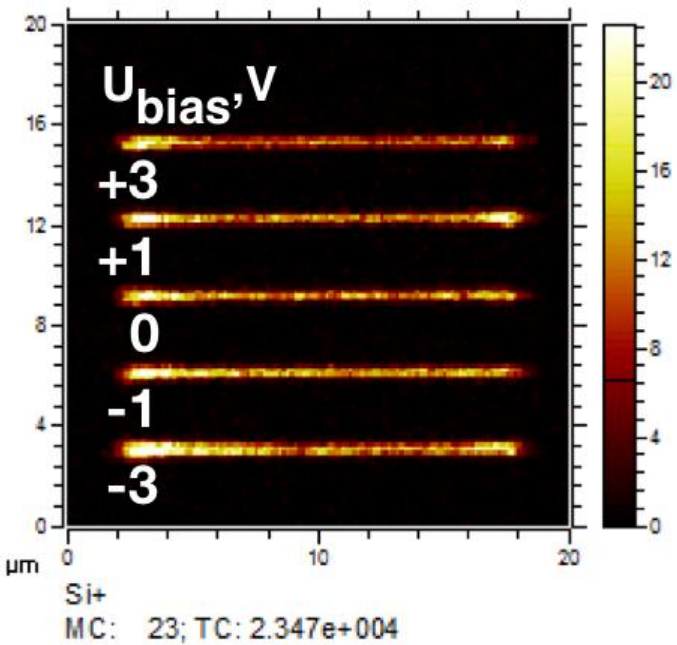

(a)

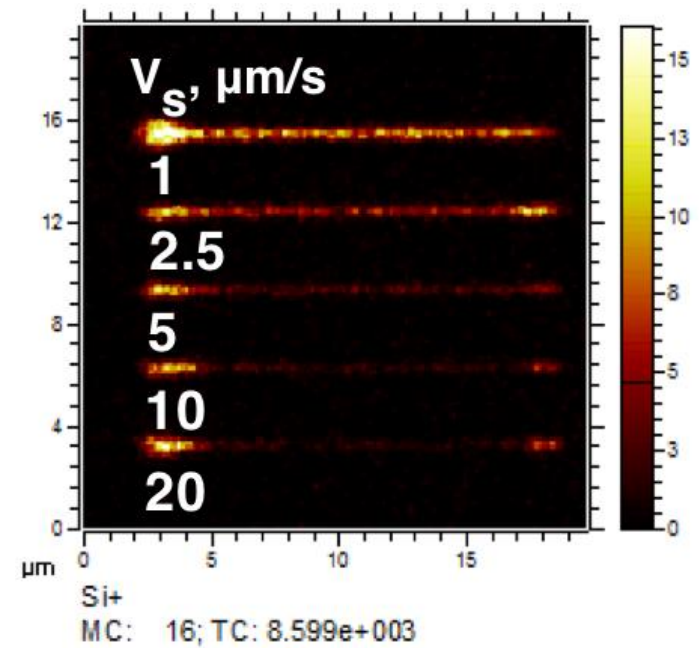

(b)

Figure 2. Line scans on PZT film surface varying tip bias (a) and scanning speed (b). 